\title{
A New Cryptanalytic Attack for PN-generators Filtered by a Boolean Function
}

\author{
Sabine Leveiller ${ }^{1,2}$, Gilles Zémor ${ }^{2}$, Philippe Guillot ${ }^{3}$, and Joseph Boutros ${ }^{2}$ \\ 1 Thales Communication \\ 66, rue du Fossé Blanc, 92231 Genevilliers, FRANCE \\ 2 Ecole Nationale Supérieure des Télécommunications \\ 46, rue Barrault, 75013 Paris, FRANCE \\ \{leveille, zemor, boutros\}@enst.fr \\ 3 Canal-Plus Technologies \\ 34, place Raoul Dautry, 75015 Paris, FRANCE \\ pguillot@canal-plus.fr
}

\begin{abstract}
We present a new cryptanalytic attack on PN-generators filtered by a Boolean function. The key-idea is to jointly combine the knowledge of the Boolean function and the LFSR structure so as to introduce a new iterative decoding technique. The results we obtained prove to be very good: indeed, even in the case of systems for which classical iterative algorithms appear powerless, our scheme enables us to recover the LFSR initial state with a very low error probability. The latter is such that ending the algorithm by an exhaustive search among sequences of limited length is hardly needed. Therefore, the overall complexity of our scheme remains very small and the algorithm appears as an excellent candidate for cryptanalysis of filtered PN-generators.
\end{abstract}

Keywords: Boolean functions, stream ciphers, APP decoding, Fourier transform.

\section{Introduction}

The original paper on correlation attacks by Meier and Staffelbach [16] together with the invention of turbo codes [2] and the rediscovery of iterative decoding $[6,23,14]$ produced an extensive effort to improve correlation attacks on stream ciphers $[10,11,3,18,12,19,20]$. These attacks are mainly based on powerful channel decoding techniques, such as iterative APP decoding of low density parity-check codes, trellis decoding (Viterbi algorithm) of large memory convolutional codes, iterative APP decoding of turbo codes, or maximum likelihood channel decoding [4]. In this paper, we address the case when the keystream is a non-linearly filtered generator [21]. Attacks on filter generators fall into two classes: deterministic attacks, mainly $[1,8,13]$, and correlation attacks. All correlation attacks to be found in the literature on such systems model the filtering Boolean function $[17,22]$ as a memoryless binary symmetric channel (BSC) [5]. Hence, all known performance results and statements are obtained in the idealized case when the 
pseudo-noise (PN) sequence [9] runs through a BSC rather than a Boolean function.

In this paper, we consider both the true structure of a filter generator without any BSC modeling and we infer the original key value (the LFSR initial state) by jointly combining the knowledge of the Boolean function and the LFSR characteristics. The linear feedback shift register (LFSR) followed by a Boolean function is illustrated in Fig.1.

This paper is organized as follows: after presenting the key-idea of the algorithm in section 2, section 3 describes the new probabilistic attack we call SOJA (Soft Output Joint Algorithm) and gives the mathematical expression of the a posteriori probabilities generated by the SOJA. Section 4 deals with a less complex but approximated version of the SOJA. In section 5 , we focus on a specific type of Boolean function, namely plateaued functions, and we analyze the performance of both algorithms. Finally, numerical results showing the performance of the SOJA attacks are given in section 6 .

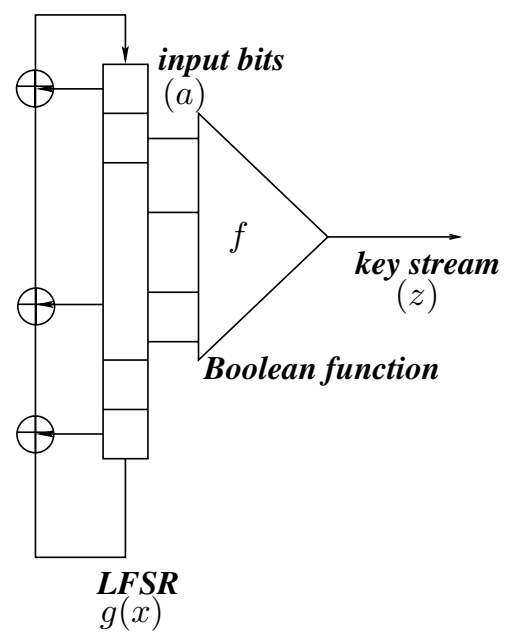

Fig. 1. The key stream filter generator

Notation. The following notation is used in this manuscript:

- $K$ is the degree of the LFSR feedback polynomial $g$ and $N$ is the length of the key-stream sequence $z$, also called received sequence in channel decoding terminology

- the original PN-sequence is denoted $a$, where $\left\{a_{t}\right\}$ are also called input bits

- $f$ denotes the $n$-input filtering Boolean function

- $X(t)=\left(X_{1}(t), \ldots, X_{n}(t)\right)$ is the input vector of the function at time $t$ 
- $\forall u \in\{0,1\}^{n}, \phi_{u}$ denotes the linear form: $\phi_{u}: x \longrightarrow(u . x)$ where $u . x=$ $\sum_{i} u_{i} x_{i}$ is the usual scalar product. Applying such a linear form to the initial sequence $(a)$ will provide us with a new sequence denoted $\left(a^{\phi_{u}}\right)$. Note that any linear form of the initial sequence satisfies the same parity-check equations as the latter.

- $\widehat{f}$ denotes the Fourier Transform of $f$ :

$$
\forall u \in\{0,1\}^{n}, \quad \widehat{f}(u)=\sum_{x \in\{0,1 \diamond n} f(x)(-1)^{u . x}
$$

- $f_{\chi}$ denotes the sign function associated to $f$ :

$$
\forall x \in\{0,1\}^{n}, f_{\chi}(x)=(-1)^{f(x)}
$$

$-r_{f}: x \longrightarrow \sum_{y}(-1)^{f(y)+f(y+x)}$ denotes the auto-correlation function

\section{An Overall View over the Proposed Algorithms}

In this section, we derive the basic and general principles of the SOJA algorithms, and we look into more details in sections 3 and 4.

The attack we propose hereafter requires the knowledge of the Boolean function table, the feedback polynomial, and a set of $d$-weight parity-check equations satisfied by the initial PN sequence. For instance, these might be provided by square elevations of the feedback polynomial as mentioned in [16] or a table lookup algorithm as described in [3], [7].

Let us introduce further notation: we call $\mathcal{E}^{b}(t)$ the set of parity-checks

$$
a_{t_{1}}+a_{t_{2}}+\ldots+a_{t_{d}}=0
$$

to which bit $a_{t}$ belongs. An element of $\mathcal{E}^{b}(t), e$, is a set of $d$ bits, among which $a_{t}$, that sum to zero. The sequence $(a)$ can be seen as a codeword of the $(N, K)$ code generated by the LFSR. This code being cyclic, its dual is also cyclic and every single parity-check shifted $n$ times gives rise to a vectorial one: let $\mathcal{E}^{v}(t)=\{E\}$ such that $E$ is a set of $d$ input vectors, $X\left(t_{1}\right), \ldots, X\left(t_{d}\right)$, among which $X(t)$, that sum to zero. Note that, if $\mu$ denotes the average cardinality of $\mathcal{E}^{v}(t)$, that is the average number of parity-checks to which $X(t)$ belongs, then the average cardinality of $\mathcal{E}^{b}(t)$ is $n \mu$.

Our joint attack consists in combining the Boolean function constraints and the information provided by PN parity-check equations to compute a posteriori probabilities $A P P\left(a_{t}\right)$ on the input bits $\left\{a_{t}\right\}$. Sections 3 and 4 propose two ways of deriving the APP's. Both these SOJA versions use the list of constraints satisfied by the $d$ vectors of every vectorial check $E$, namely

$-X\left(t_{1}\right)+\ldots+X\left(t_{d}\right)=0_{n}$

- their image by the Boolean function $f$ is the received $d$-tuple $z_{t_{1}}, \ldots, z_{t_{d}}$ 
$0_{n}$ being the all-zero vector.

Hence, we have a $d \times n$ constrained system written as:

$$
\begin{aligned}
z_{t_{1}} & =f\left(X_{1}\left(t_{1}\right), X_{2}\left(t_{1}\right), \ldots, X_{n}\left(t_{1}\right)\right) \\
z_{t_{2}} & =f\left(X_{1}\left(t_{2}\right), X_{2}\left(t_{2}\right), \ldots, X_{n}\left(t_{2}\right)\right) \\
\ldots & \\
z_{t_{d}} & =f\left(X_{1}\left(t_{d}\right), X_{2}\left(t_{d}\right), \ldots, X_{n}\left(t_{d}\right)\right) \\
\text { with } \quad 0_{n} & =\sum_{i} X\left(t_{i}\right)
\end{aligned}
$$

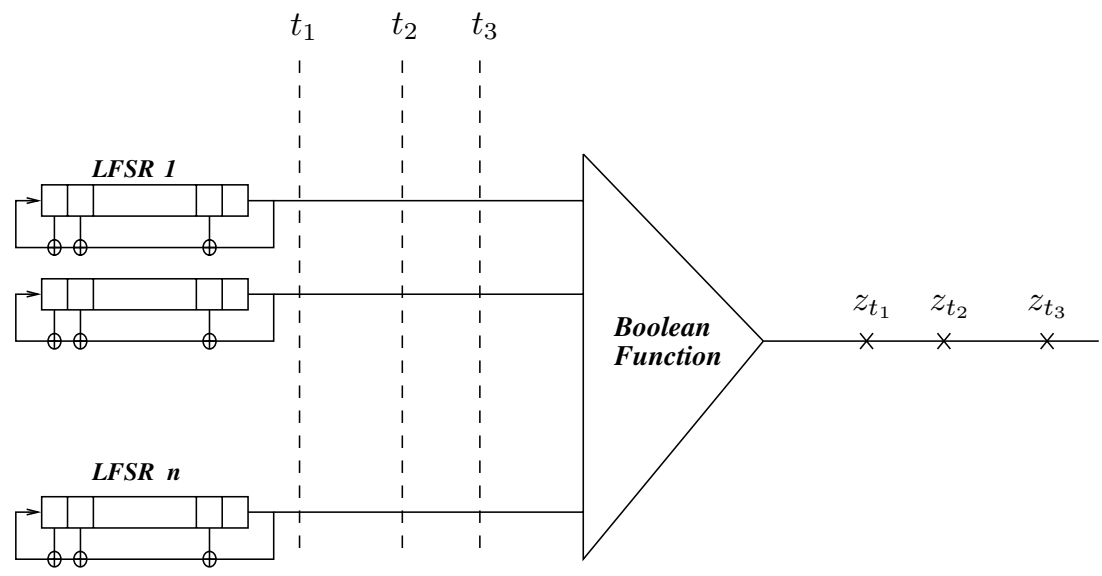

Fig. 2. Alternative representation of the key stream generator with $X\left(t_{1}\right)+$ $X\left(t_{2}\right)+X\left(t_{3}\right)=0$

To illustrate our purpose, Fig.2 gives an equivalent, but more appropriate representation of Fig.1 with a weight-3 parity-check equation: $X\left(t_{1}\right)+X\left(t_{2}\right)+$ $X\left(t_{3}\right)=0$. The $\mathrm{PN}$ generator is replaced by $n$ parallel $\mathrm{PN}$ generators with identical feedback polynomials but different initial states. The 3 input vectors, $X\left(t_{1}\right)=\left(X_{1}\left(t_{1}\right), \ldots, X_{n}\left(t_{1}\right)\right), X\left(t_{2}\right)=\left(X_{1}\left(t_{2}\right), \ldots, X_{n}\left(t_{2}\right)\right), X\left(t_{3}\right)=$ $\left(X_{1}\left(t_{3}\right), \ldots, X_{n}\left(t_{3}\right)\right)$ sum to zero, while their images through $f$ are $z_{t_{1}}, z_{t_{2}}$, and $z_{t_{3}}$ respectively.

Basically the general principle of the SOJA is to use the knowledge of the Boolean function jointly with vectorial parity-checks to estimate $P(X(t)=x)$ that is further used to get the APP probabilities $\Lambda_{\phi_{u}}(t)=P_{a p p}\left(a_{t}^{\phi_{u}}\right)$, where $a_{t}^{\phi_{u}}$ is a linear form of the input sequence that is chosen according to the Boolean function properties. 
The overall decoding algorithm can be written in the following way:

1. Inputs: $g(X), f,\left\{\mathcal{E}^{v}(t)\right\}$ and $\backslash$ or $\left\{\mathcal{E}^{b}(t)\right\},(z)$

2. Processing steps:

- Generate the APP's using SOJA-1 (see section 3) or SOJA-2 ( see section 4).

- Once the APP's are computed, cryptanalyze the system using either SOJA-Gallager or SOJA-threshold described hereafter.

3. Output : initial state of the $\mathrm{PN}$ generator.

Let us describe both decoding strategies in more details:

- SOJA-Gallager: iterative decoding using these SOJA APPs as channel observations.

We briefly recall a simplified version of Gallager's probabilistic decoding algorithm [6]: let $A P P^{\theta}\left(a_{t}^{\phi_{u}}\right)$ be the $\mathrm{APP}$ of bit $a_{t}^{\phi_{u}}$ at iteration $\theta$ produced by the probabilistic decoder, and $\operatorname{Obs}\left(a_{t}^{\phi_{u}}\right)$ be the channel observation. Assuming that for all $t$, the bits among $\mathcal{E}_{b}^{t}$ are statistically independent, the simplified Gallager algorithm is:

1. $\theta=0: \forall t \in[1, N], \quad A P P^{(0)}\left(a_{t}^{\phi_{u}}\right)=\operatorname{Obs}\left(a_{t}^{\phi_{u}}\right)=\Lambda_{\phi_{u}}(t)$

2. $\theta>0: \forall t \in[1, N]$,

$$
\begin{aligned}
& A P P^{(\theta)}\left(a_{t}^{\phi_{u}}\right) \propto \operatorname{Obs}\left(a_{t}^{\phi_{u}}\right) \times \prod_{e \in \mathcal{E}_{t}^{b}} \operatorname{Prob}\left(a_{t}^{\phi_{u}}=1 \text { in } e \text { at iteration } \theta\right)
\end{aligned}
$$

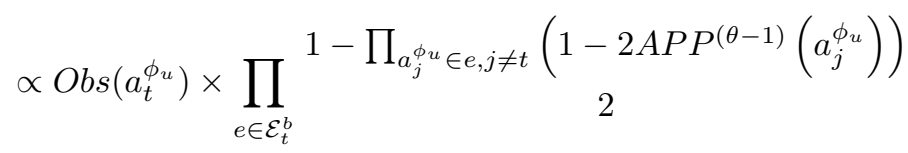

3. If $A P P^{\theta_{\max }}\left(a_{t}^{\phi_{u}}\right)>\frac{1}{2}, a_{t}^{\phi_{u}}$ is decoded as 1 , else $a_{t}^{\phi_{u}}$ is decoded as 0 .

where $\propto$ stands for "proportional to" (the proportionality factor depending on the considered bit). Steps (2) and (3) include a normalization phase of the $A P P$ of each bit so that $\forall t, \forall \theta, A P P^{(\theta)}\left(a_{t}^{\phi_{u}}=1\right)+A P P^{(\theta)}\left(a_{t}^{\phi_{u}}=0\right)=1$. The above algorithm is a simplification of the initial Gallager decoder, since it doesn't handle extrinsic information [2]. The incoming a priori probability is taken to be equal to the previous APP.

- SOJA-Threshold: a threshold decoding on SOJA APPs.

The a posteriori probability $0 \leq \Lambda\left(a_{t}^{\phi_{u}}\right) \leq 1$ produced by the SOJA is considered to be reliable when its value is close to 0 or 1 and unreliable if $\left|\Lambda\left(a_{t}^{\phi_{u}}\right)-0.5\right|$ is small. Hence, we can select the most reliable bits, decode them by comparing their APP with $\frac{1}{2}$, and invert the PN sequence system so as to provide the initial state of the PN generator. Finally, we check that the solution is correct by re-encoding the sequence.

To apply this strategy, we select the $K$ most reliable and linearly independent bits and we proceed as follows: 
- if $\Lambda\left(a_{t}^{\phi_{u}}\right)-0.5>0, a_{t}^{\phi_{u}}$ is decoded as 1 ,

- if $0.5-\Lambda\left(a_{t}^{\phi_{u}}\right)>0, a_{t}^{\phi_{u}}$ is decoded as 0 .

\section{New Probabilistic Attack: Soft Output Joint Attack, SOJA-1}

\subsection{Algorithm Description}

A restricted set of input vectors jointly satisfy the constrained system (1) and this provides accurate information on the input vectors: for a given output $d$-tuple $z_{t_{1}}, \ldots, z_{t_{d}}$, we can enumerate all vectors in the truth table of the function $f$ whose image matches the output (first constraint), and that sum to zero (second constraint). Let us define the set $z^{\mathcal{E}}$ of all observed bits $z_{s}$ such that $X(s)$ is in some vectorial check $E$ of $\mathcal{E}^{v}(t)$ and $z^{E}$ the bits $z_{s}$ such that $s$ belongs to the support of $E$, i.e. $z^{\mathcal{E}}=\bigcup_{E \in \mathcal{E}^{v}(t)} z^{E}$. We also define:

$$
\Gamma^{t}(x)=P\left(X(t)=x \text { in } E \mid z^{E}, f\right)
$$

as the proportion of arrays (see (1) ) such that $X(t)=x ; x \in\{0,1\}^{n}$ and $E$ is a vectorial parity-check in $\mathcal{E}^{v}(t)$. Assuming that the only vector common to every pair of elements of the set $\mathcal{E}^{v}(t)$ is precisely $X(t)$, that is, assuming $\mathcal{E}^{v}(t)$, seen as a Tanner graph, doesn't contain any cycle of length 4 , we can write:

$$
\forall t, P\left(X(t)=x \mid z^{\mathcal{E}}, f\right)=\frac{\prod_{E \in \mathcal{E}^{v}(t)} \Gamma^{t}(x)}{\sum_{y} \prod_{E \in \mathcal{E}^{v}(t)} \Gamma^{t}(y)}
$$

and then $\forall u \in\{0,1\}^{n}$

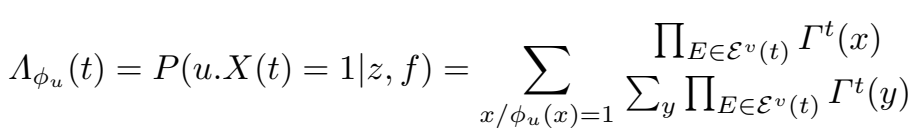

As noted before, according to the function properties, we might decode a linear form of the input sequence.

\subsection{Mathematical Expression of SOJA-1}

Let us derive the expression of $\Gamma^{t}$ in more details.

The only assumption that we make on the Boolean function is that it is balanced. The particular case of plateaued functions is viewed later in section 5 .

Proposition 1. Given a received d-tuple $\left(z_{1}, \ldots, z_{d}\right)$, we can write

$$
\Gamma(x)=1 \boldsymbol{q}_{\boldsymbol{q} f(x)=z_{1} \diamond}(x) \times \begin{gathered}
2^{(d-1) n+1}+(-1)^{\sum_{i=2}^{d} z_{i}} 2 . \widehat{g}(x) \\
2^{d n}+(-1)^{\sum_{i=1}^{d} z_{i}} \widehat{h}(0)
\end{gathered}
$$

where $\Gamma$ was defined in (2) (the time index is omitted for the sake of simplicity), $g={\widehat{f_{\chi}}}^{d-1}$ and $h={\widehat{f_{\chi}}}^{d}$. 
Proof. See Appendix A, prop. 1.

The above expression deals with the antecedent of $z_{1}$, but it holds for the other input vectors by permuting them in the formula. This expression also shows that the quantity $\Gamma$ needs to be computed for all $x \in\{0,1\}^{n}$ and for a given parity of $\sum_{i=2}^{d} z_{i}$. The most efficient way to proceed is then to build a table in which we store the values of $\Gamma$; assuming the function is balanced, we must store $2^{n}$ values. Each computation requires at most $\mathcal{O}\left(n 2^{n+1}\right)$ elementary operations. Recall that $\mu$ is the average cardinality of $\mathcal{E}^{v}(t)$; the overall complexity of the computation of the APP's is in $\mathcal{O}\left(n 2^{2 n+1}+N \mu 2^{n-1}\right)$.

\section{Remarks}

- When the weight of the parity-checks equals 3, we get

$$
\left.\Gamma(x) \propto 2^{n}+(-1)^{z_{2}+z_{3}} r_{f}(x)\right]
$$

The value of $\Gamma(x)$ is then directly related to the auto-correlation function associated to $f$.

Moreover the case when $d=3$ is very interesting because it enables us to detect the all-zero vector with a very high probability. More precisely, let us consider one of the checks to which $X$ belongs and let us write it as: $X+Y_{2}+Y_{3}=0_{n}$. Assume $X=0_{n}$; then $Y_{2}=Y_{3}$ and the corresponding received bits are equal, that is $z_{2}=f\left(Y_{2}\right)=f\left(Y_{3}\right)=z_{3}$. The interesting point is that this equality also stands in all other equations in which $X$ is involved, as depicted on Fig. 3. From the cryptanalyst's point of view, such an equality between two bits within all parity-checks is very rare: if $\mu$ denotes the cardinality of the set of equations of $X$, and if the function $f$ takes the values 0 and 1 equiprobably, the probability of such an event is in $1 / 2^{\mu}$. Therefore, using the SOJA is a very efficient way to detect the event of having the all-zero vector at the input. This can be extended to parity-checks of higher weight if cycles among the equations can be found.

- Earlier, we mentioned the fact that, according to the properties of the Boolean function, we could try to decode a particular linear form of the input sequence. However, when the spacings between the function inputs are coprime, decoding the "one-weight" linear form of the type $\phi_{i}: x \longrightarrow x_{i}$ often guarantees much better performance. As a matter of fact, these linear forms are the only ones that can be "shifted", i.e. which are involved in $n$ input vectors successively on each component of the input, while each bit of a non-trivial linear form of the input is involved in the generation of only one output bit. Then instead of having one quantity $\Lambda_{\phi_{u}}(t)$ associated to one bit of the sequence to be decoded, we have $n$ quantities.

Next we propose a lower complexity SOJA algorithm, which actually results from an approximation of the above version. 


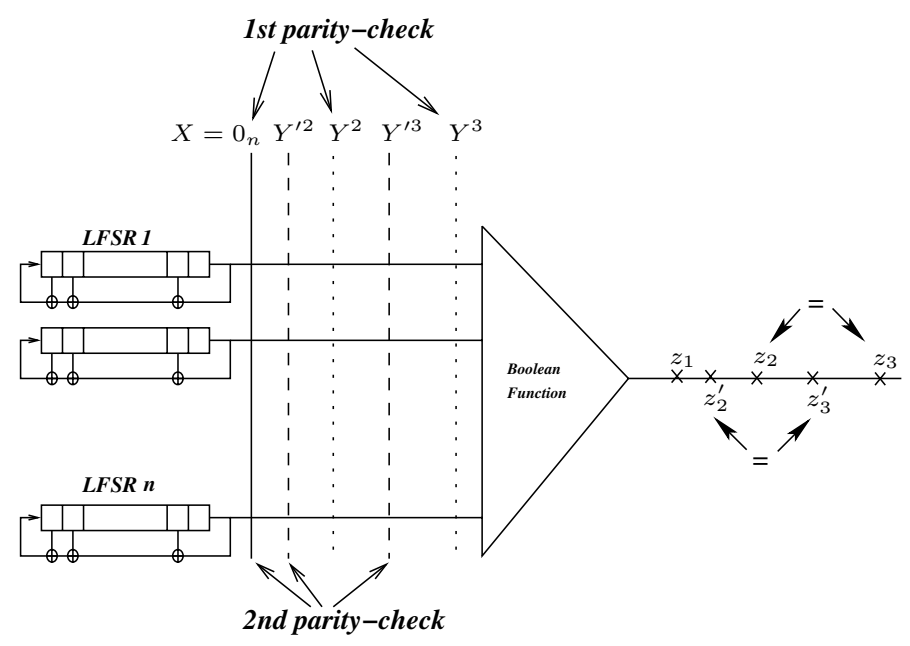

Fig. 3. Detection of the all-zero vector in the particular case of 3-weight parity checks

\section{An Approximated SOJA Version, SOJA-2}

The approximated version of the SOJA that we propose hereafter relies on the assumption that the bits among an input vector are independent: from a parity check, we directly deduce informations on the vector components of the sequence to be decoded, and, for a given bit, the scalar probabilities brought by all its parity-checks are multiplied together as if they were independent. This new scheme appears less complex than SOJA-1, but its major drawback is to give poor results when applied to non-trivial linear forms of the input sequence. Therefore, we will only consider the case when one strives to decode the original sequence $(a)$, and not a linear form of it.

A paradox is that this simplified scheme works best when the function is resilient that is when the independence assumption of the input bits is the most justified.

\subsection{Algorithm Description}

The basic principle is identical to that of the SOJA-1: considering a parity-check $X\left(t_{1}\right)+X\left(t_{2}\right)+\ldots+X\left(t_{d}\right)=0_{n}$, we enumerate the input vectors that jointly satisfy the parity-check and correspond to the received bits $z_{t_{1}} \ldots z_{t_{d}}$. Then, on each components, we can evaluate the proportions of $1 \mathrm{~s}$ and $0 \mathrm{~s}$, and we deduce probabilities on each of these components; we obtain individual a posteriori probabilities on the input bits: for all $m, j$, one computes:

$$
\begin{aligned}
\mathcal{A}_{j, m} & =P\left(X_{m}\left(t_{j}\right)=1 \mid \forall i \in[1, d] f\left(X\left(t_{i}\right)\right)=z_{t_{i}} \text { and } X\left(t_{1}\right)+\ldots+X\left(t_{d}\right)=0_{n}\right) \\
& =P\left(X_{m}\left(t_{j}\right)=1 \mid \forall i \in[1, d-1] f\left(X\left(t_{i}\right)\right)=z_{t_{i}}, f\left(\sum_{\ell=0}^{d-1} X\left(t_{\ell}\right)\right)=z_{t_{d}}\right)
\end{aligned}
$$


$m \in[1, n], j \in[1, d]$.

This quantity represents the partial APP of the bit that coincides with the $m$-th component of the antecedent of $z_{t_{j}}$. We notice that this probability only depends on the value of the vector $\left(z_{t_{1}}, \ldots, z_{t_{d}}\right)$; therefore the computation of these partial APPs is made once for every $2^{d}$ possible $d$-tuples, and stored in a $2^{d} \times d \times n$ table. Another simplification is to be noted: the computations have to be done for each $\left(z_{t_{1}}, \ldots, z_{t_{d}}\right)$ of a given weight, and the rest of the table is completed by appropriate permutations on the input. Then, using this table, the received vectors that correspond to each parity-check equation will provide additional information on the components of their antecedents.

Assuming that the information brought by all equations are independent, we get the a posteriori probabilities, $\Lambda$, generated by the SOJA-2, namely:

$$
\forall t \in[1, N], \quad \Lambda\left(a_{t}=1\right) \propto \prod_{e \in \mathcal{E}^{s}(t)} \mathcal{A}_{j, m}
$$

Note that the superscript $\phi_{u}$ is omitted because, as noted before, SOJA-2 is only used to decode trivial linear forms of weight one. Once these APP's are evaluated, we suggest to apply one of the two decoding strategies presented in section 2 .

We will now derive a mathematical expression of the above probabilities.

\subsection{Mathematical Expression of SOJA-2}

Let $v=\left(0, \ldots, 0, v_{m}=1,0, \ldots\right) \in\{0,1\}^{n}$, where $v$ is a pointer at the $m$-th input of the Boolean function, i.e. the $m$-th canonical vector.

For a given received $d$-tuple $\left(z_{1}, \ldots, z_{d}\right)$, we define the correlation coefficient, for $1 \leq j \leq d$,

$$
c^{j}(v)=\frac{\Omega^{j}(v)}{\Omega^{j}\left(0_{n}\right)}=2 \mathcal{A}_{j, m}-1
$$

where

$$
\begin{aligned}
\Omega^{j}(v)=\sum_{Y^{1}} & \sum_{Y^{2}} \cdots \sum_{Y^{d-1}} \mathbb{1}_{\boldsymbol{q}_{f f}\left(Y^{1}\right)=z_{1} \diamond}\left(Y^{1}\right) \mathbb{1}_{\boldsymbol{\Phi}_{f}\left(Y^{2}\right)=z_{2} \diamond}\left(Y^{2}\right) \cdots \\
& \times \mathbb{1}_{\boldsymbol{\Psi}_{f\left(\sum_{\ell=1}^{d-1} Y^{\ell}\right)=z_{d} \diamond} \diamond}\left(\sum_{\ell=1}^{d-1} Y^{\ell} \quad(-1)^{v . Y^{j}}\right.
\end{aligned}
$$

and $0_{n}$ is the all-zero vector, and $\forall \ell, Y^{\ell} \in\{0,1\}^{n}$. Developing the above expressions, we can state the following proposition:

Proposition 2. For a given received d-tuple $\left(z_{1}, \ldots, z_{d}\right), \Omega^{j}(v)$ is given by

$$
\begin{aligned}
\Omega^{j}(v)= & \frac{1}{2^{d} 2^{n}} \sum_{s}\left[2^{n} 1_{\boldsymbol{\Phi}_{s=v} \diamond}(s)+(-1)^{z_{j}} \widehat{f_{\chi}}(s+v)\right] \\
& \times \prod_{\ell \neq i}\left[2^{n} 1_{\boldsymbol{q}_{s=0_{n} \diamond}}(s)+(-1)^{z_{\ell}} \widehat{f_{\chi}}(s)\right]
\end{aligned}
$$


If we further assume that $f$ is balanced, and correlation immune (i.e. $f$ is resilient) whatever its nonzero order of resiliency, $c^{j}(v)$ takes the very simple expression:

$$
c^{j}(v)=\begin{gathered}
(-1)^{\sum_{\ell} z_{\ell}} \sum_{s} \widehat{f_{\chi}}(v+s){\widehat{f_{\chi}}}^{d-1}(s) \\
2^{d n}+(-1)^{\sum_{\ell} z_{\ell}} \widehat{h}(0)
\end{gathered}
$$

where $h={\widehat{f_{\chi}}}^{d}$.

As noted before, it clearly appears that the value of $c^{j}(v)$ needs only to be computed for a given weight of the output vector: all the other probabilities can be derived by appropriate permutations. When the function $f$ is resilient as in (7), the correlation coefficient $c^{j}(v)$ depends on the parity of the output vector and it does not depend on the time position $j$.

Moreover, expression (6) enables a quick evaluation of the SOJA complexity: in general, for any $f$, each correlation roughly takes $n \times 2^{n}$ computation steps in the Fourier transform. Therefore, the overall complexity is: $\mathcal{O}\left(n \times 2^{n}+N n \mu\right)$.

\section{The Effect of Plateaued Functions}

We have tried to envisage what Boolean functions $f$ would make our attack least effective. In [24], Zheng and Zhang introduced the notion of plateaued functions: a function $f$ is said to be plateaued iff

$$
\exists r \text { such that } \forall u,{\widehat{f_{\chi}}}^{2}(u)=0 \text { or } 2^{2 n-r}
$$

From the cryptanalyst's point of view, such functions are very embarrassing, because the linear forms of the initial sequence that have non-zero input-output correlation are all equidistant from the keystream $z$ (considering the complement of the received sequence $z$ when the correlation is negative). Therefore, a BSC decoder will tend to oscillate between them. We will show that using plateaued functions affects our SOJA algorithm performance when the parity-checks are even weight.

\subsection{The Effect of Plateaued Functions on the SOJA-1}

Proposition 3. If the function $f$ is plateaued and the parity-checks are oddweighted, we have

$$
\Gamma(x)=\left[1+(-1)^{f(x)+z_{1}}\right]^{2^{(d-1) n}+(-1)^{\sum_{2}^{d} z_{i}} \times 2^{(2 n-r)(p-1)} r_{f}(x)} \begin{gathered}
2^{d n}+(-1)^{\sum_{1}^{d} z_{i}} \times 2^{(2 n-r) p+n} f_{\chi}(0)
\end{gathered}
$$

where $d=2 p+1$, and $S p\left(f_{\chi}\right)=\left\{0, \pm 2^{n-\frac{r}{2}}\right\}$.

Proof. See Appendix A, prop. 3.

Proposition 4. If the function is plateaued and if the parity-checks are evenweighted, we don't gain anything over the classical initialization, which uses the function non-zero correlations .

Proof. See Appendix A, prop.4. 


\subsection{Effect of Plateaued Resilient Functions on SOJA-2}

Proposition 5. For any plateaued function $f$,

$$
\forall(p, q) \in \mathbb{N}, \forall v \neq 0_{n} \in\{0,1\}^{n}, \quad \sum_{s}{\widehat{f_{\chi}}}^{2 p+1}(v+s){\widehat{f_{\chi}}}^{2 q+1}(s)=0
$$

Proof. See Appendix A, prop. 5.

Corollary 1. If $f$ is a plateaued resilient Boolean function, only odd-weight parity-check equations are usable when applying the SOJA.

If $f$ is resilient, the correlation values are given by equation (7). Then, if the parity-check equation weight is even, by applying the above proposition with $d=2 p$ and $q=0$, we find that all correlations are zero.

\section{Results and Comments}

\subsection{The Experiment}

We present two different sets of results, obtained with the two resilient plateaued Boolean functions written below.

For each function, we compare:

- classical simplified Gallager iterative decoding (see the first decoding strategy in section 2) applied to $z$ with the Boolean function modeled as a BSC.

- classical simplified Gallager iterative decoding applied to a genuine BSC with the same transition probability; this is to underline the limitations of BSC modeling.

- (SOJA-1)-Gallager algorithm, (SOJA-1)- threshold algorithm.

- (SOJA-2)-Gallager algorithm, (SOJA-2)- threshold algorithm.

When the Boolean function is considered, the spacings between its input are taken to be coprime. Moreover, in both cases (SOJA-1 and SOJA-2), we chose to decode the trivial linear form that corresponds to a single input of the Boolean function, because, as noted before, when the spacings are coprime, the latter is the only linear form that is involved in $n$ output bits.

The results are summarized in tables 1 and 2 . For different values of the length $N$ of observation $z$, we present the results obtained with the above decoding schemes. The second and third rows feature the average success percentage of Gallager decoding with a BSC channel (simplest model) and a Boolean function respectively (with the same corresponding transition probability). The last four rows contain the results obtained with our algorithms: in the fourth row we present the average success of the (SOJA-1)-Gallager algorithm and in the fifth one, the results obtained when using (SOJA-2)-algorithm. The last two rows contain the results obtained with the SOJA-threshold algorithms, SOJA-1 and SOJA-2, that is the average error rate among the $K$ decoded bits, $\rho_{a v}$. 
First Application In this section, the results are obtained with the 3-resilient 7-input plateaued function

$$
\begin{gathered}
f(x)=1+x_{1}+x_{2}+x_{3}+x_{4}+x_{5}+x_{6}+x_{1} x_{7}+x_{2}\left(x_{3}+x_{7}\right)+ \\
x_{1} x_{2}\left(x_{3}+x_{6}+x_{7}\right)
\end{gathered}
$$

with $r=4$ that is equivalent transition probability $p=0.375$. The polynomial is $g(x)=1+X+X^{3}+X^{5}+X^{9}+X^{11}+X^{12}+X^{17}+X^{19}+X^{21}+X^{25}+X^{27}+X^{29}+$ $X^{32}+X^{33}+X^{38}+X^{40}$, and the weight-5 parity-check equations are obtained with the algorithm developed in [3]. Moreover the spacings between the inputs of the Boolean function are taken to be coprime. The results are presented in table 1.

$$
\begin{array}{c|c|c|}
\text { Decoding strategy } & \mathbf{N}=\mathbf{1 1 0 0 0} & \mathbf{N}=\mathbf{1 7 0 0 0} \\
\text { real BSC }(p=0.375)+\text { Gallager } & \text { no convergence } & 85 \% \\
f \text { modeled as a BSC + Gallager } & \text { no convergence } & 19 \% \\
f+(\text { SOJA-2)-Gallager } & \text { no convergence } & 36 \% \\
f+(\text { SOJA-1)-Gallager } & \text { no convergence } & 100 \% \\
f+(\text { SOJA-2)-threshold } & \rho_{a v}=0.226 & \rho_{a v}=0.02 \\
f+\text { (SOJA-1)-threshold } & \rho_{a v}=0.096 & \rho_{a v}<10^{-4}
\end{array}
$$

Table 1. $\mathrm{K}=40$, 7-input 3-resilient plateaued function

Second Application Here, we consider the system where the PN-sequence is generated by $g(X)=1+X^{37}+X^{100}$ and filtered by the 8-input 2-resilient plateaued function

$$
\begin{aligned}
f(x)=x_{1} & +x_{4}+x_{5}+x_{6}+x_{7}+x_{1}\left(x_{2}+x_{7}\right)+x_{2} x_{6}+x_{3}\left(x_{6}+x_{8}\right) \\
& +x_{1} x_{2}\left(x_{4}+x_{6}+x_{7}+x_{8}\right)+x_{1} x_{3}\left(x_{2}+x_{6}\right)+x_{1} x_{2} x_{3}\left(x_{4}+x_{5}+x_{8}\right)
\end{aligned}
$$

with $r=6$, that is nonzero correlation equal to $c=\frac{1}{8}$ or equivalent transition probability in the BSC representation equal to $p=0.4375$. We worked with weight-3 parity-check equations, obtained by successive square elevations of the weight- 3 feedback polynomial. The spacings between the Boolean function are taken coprime and span the whole memory of the PN generator. The corresponding results are featured in table 2 .

\subsection{Comments on the 3 Variations on Gallager Decoding}

We mentioned the results obtained with Gallager simplified iterative decoding applied in different situations. It clearly appears in table 1 that iterative algorithms converge worst when applied to a Boolean function rather than to the corresponding BSC model (with same transition probability). The function we used is a plateaued function, which might not act in favor of iterative decoding 


\begin{tabular}{|c|c|c|c|}
$\begin{array}{c}\text { Decoding strategy } \\
\text { real BSC model }(\mathbf{p}=0.4375)\end{array}$ & $\mathbf{N}=\mathbf{5 0 0 0}$ & $\mathbf{N}=\mathbf{1 1 0 0 0}$ & $\mathbf{N}=\mathbf{3 0 0 0 0}$ \\
+ Gallager & & & \\
$f$ modeled as a BSC & no convergence & no convergence & no convergence \\
+ Gallager & & & \\
$f+$ (SOJA-2)-Gallager & no convergence & no convergence & no convergence \\
$f+$ (SOJA-1)-Gallager & $1 \%$ & $42 \%$ & $86 \%$ \\
$f+$ (SOJA-2)-threshold & $\rho_{a v}=0.245$ & $\rho_{a v}=0.165$ & $\rho_{a v}=0.08$ \\
$f+$ (SOJA-1)-threshold & $\rho_{a v}=0.004$ & $\rho_{a v}<10^{-4}$ & $\rho_{a v}<10^{-4}$
\end{tabular}

Table 2. K=100, 8-input 2-resilient plateaued function

as all non-zero correlations are equal in absolute value. Note however that even (SOJA-2)-Gallager improves over classical Gallager iterative decoding.

In the second application, table 2 shows that classical iterative decoding doesn't work in the considered situation. We even tried higher values of $N$ (up to 200,000) and didn't observe any convergence. Recall that in this particular example, the parity-check equations were obtained by successive squarings of the (low-weight) feedback polynomial. Unlike the table lookup algorithm [3] (which would require unreasonable complexity to be applied here), this provides very few parity check equations, not enough to enable iterative algorithm to converge.

Finally, it is to be noted that the approximated version of the SOJA, SOJA-2, doesn't give as good results as the true version: while in the first example, a slight increase of the length $N$ of the received sequence tends to justify the utility of SOJA-2 the performance of the latter is far behind those of SOJA-1 in the second example, specially as regards SOJA-Gallager. A trade-off must be found between the system parameters and the available computational ability.

\subsection{The Efficiency of SOJA-Threshold}

In both applications, the SOJA-threshold algorithm appears as the most efficient way to decrypt the system. In some cases when most iterative algorithms don't converge, the SOJA-threshold gives good results.

The most striking result is obtained in the second application: the SOJA-threshold gives significantly better results than other algorithms with very few required bits and enables us to recover the initial state of the sequence with a complexity which is much lower than that of the SOJA-Gallager. One might expect that the SOJA-threshold decoding should be followed by an exhaustive search among all sequences of $K$ bits to correct the wrongly decoded bits. Yet, as illustrated in both tables, a slight increase of $N$ enables to lower significantly the remaining error rate. The global complexity of the SOJA is therefore limited.

\section{Conclusion}

In this paper, we derived a new algorithm for filtered LFSR cryptanalysis: our attack jointly takes advantage of the knowledge of both the Boolean function and 
the LFSR structure to provide better information on the input bits. Next, we proposed two decoding strategies, and surprisingly, the less complex is very often the most efficient one. We obtained very good results and extend the range of cryptanalysis to systems for which Gallager's algorithm cannot hope to converge, mostly because the Boolean function is plateaued and because of the lack of parity-check equations when the degree of the feedback polynomial is too high. However, if the SOJA gives better results than more usual iterative algorithms, it must be pointed out that this algorithm needs low-weight parity-checks to be processed which is an important issue; still, the number of parity-checks required for convergence is not as high as the number required for classical iterative algorithms. Besides parity-checks, the properties of the Boolean function also play an important part: it would be interesting to derive criterions on Boolean functions that would describe their resistance to such attacks.

\section{References}

1. R.J. Anderson, "Searching for the optimum correlation attack," Fast Software Encryption -Leuven 94, Lectures Notes in Computer Science, vol. 1008, pp. 137-143, Springer-Verlag, 1995.

2. C. Berrou, A. Glavieux and P. Thitimajshima: "Near Shannon limit error-correcting coding and decoding: turbo-codes," Proceedings of ICC'93, Geneva, pp. 1064-1070, May 1993.

3. A. Canteaut and M. Trabbia: "Improved fast correlation attacks using parity-check equations of weight 4 and 5," Advances in Cryptology - EUROCRYPT 2000, Lecture Notes in Computer Science, vol. 1807, pp. 573-588, Springer Verlag, 2000.

4. V. V. Chepyzhov, T. Johansson and B. Smeets, " A simple algorithm for fast correlation attacks on stream ciphers," Fast Software Encryption 2000, Lecture Notes in Computer Science, vol. 1978, pp. 181-195, Springer Verlag, 2001.

5. T.M. Cover and J.A. Thomas: Elements of information theory. Wiley series in Telecommunications, 1991.

6. R.G. Gallager: Low Density Parity check codes. MIT Press, Cambridge, MA, 1963.

7. J.Dj. Golic, "Computation of low-weight parity-check polynomials," Electronics Letters, vol. 32, pp. 1981-1982, 1996.

8. J.Dj. Golic, A. Clark and E. Dawson, "Generalized Inversion Attack on Nonlinear Filter Generators," IEEE Transactions on computers, vol.49, No. 10, October 2000.

9. S.W. Golomb: Shift register sequences. Holden-Day, San Francisco, 1967.

10. T. Johansson and F. Jönsson: "Improved fast correlation attack on stream ciphers via convolutional codes," Advances in Cryptology - EUROCRYPT'99, Lecture Notes in Computer Science, vol. 1592, pp. 347-362, Springer Verlag, 1999.

11. T. Johansson and F. Jönsson: "Fast correlation attacks based on turbo code techniques," Advances in Cryptology - CRYPTO'99, in Lecture Notes in Computer Science, vol. 1666, pp. 181-197, Springer Verlag, 1999.

12. T. Johansson and F. Jönsson, "Fast correlation attacks through reconstruction of linear polynomials," Advances in Cryptology - CRYPTO'2000, Lecture Notes in Computer Science, vol. 1880, pp. 300-315, Springer Verlag, 2000.

13. S. Leveiller, J.J. Boutros, P. Guillot, G. Zémor, "Cryptanalysis of nonlinear filter generators with $\{0,1\}$-metric Viterbi decoding," IMA International Conference 2001, Lecture Notes in Computer Science, vol. 2260, pp. 402-414, Springer Verlag, 2001. 
14. D.J.C MacKay: "Good Error-Correcting Codes based on Very Sparse Matrices," IEEE Transactions on Information Theory, vol. 45, March 1999.

15. J.L. Massey: Threshold Decoding. MIT Press, Cambridge, MA, 1963.

16. W. Meier and O. Staffelbach: "Fast correlation attack on certain stream ciphers," Journal of Cryptology, pp. 159-176, 1989.

17. W. Meier and O. Staffelbach: "Nonlinearity Criteria for Cryptographic Functions," Advances in Cryptology - EUROCRYPT'89, Lecture Notes in Computer Science, vol. 434, pp. 549-562, Springer Verlag, 1990.

18. M.J. Mihaljević, M.P.C. Fossorier and H. Imai, "On decoding techniques for cryptanalysis of certain encryption algorithms", IEICE Transactions on Fundamentals, vol. E84-A, pp. 919-930, Apr. 2001.

19. M.J. Mihaljević, M.P.C. Fossorier and H. Imai, "A low-complexity and highperformance algorithm for the fast correlation attack", Fast Software Encryption 2000, Lecture Notes in Computer Science, vol. 1978, pp. 196-212, Springer Verlag, 2001.

20. M.J. Mihaljević, M.P.C. Fossorier and H. Imai, "Fast correlation attack algorithm with the list decoding and an application", Fast Software Encryption 2001, Lecture Notes in Computer Science, vol. 2355, pp. 196-210, Springer Verlag, 2002.

21. R.A. Rueppel: Analysis and Design of Stream Ciphers. Berlin: Springer-Verlag, 1986.

22. T. Siegenthaler: "Correlation-Immunity of Nonlinear Combining Functions for Cryptographic Applications," IEEE Transactions on Information Theory, vol. IT30, pp. 776-780, 1984.

23. R.M. Tanner: "A recursive approach to low complexity codes," IEEE Transactions on Information Theory, vol. IT-27, Sept 1981.

24. Y. Zheng and X.-M. Zhang: "Plateaued Functions," 2nd International Conference on Information and Communications Security, ICISC'99, Lecture Notes in Computer Science, vol. 1758, pp. 284-300, Springer-Verlag, 1999.

\section{Appendix A}

Let us recall that the basic assumption made upon the Boolean function is its balanceness.

Proposition 1 Given a received d-tuple $\left(z_{1}, \ldots, z_{d}\right)$, we can write

$$
\Gamma(x)=1 \operatorname{l}_{\boldsymbol{q}_{f}(x)=z_{1} \diamond}(x) \times \begin{gathered}
2^{(d-1) n+1}+(-1)^{\sum_{i=2}^{d} z_{i}} 2 . \widehat{g}(x) \\
2^{d n}+(-1)^{\sum_{i=1}^{d} z_{i}} \widehat{h}(0)
\end{gathered}
$$

where $\Gamma, g$, and $h$ were defined in (2).

Proof. Let us define

$$
\begin{aligned}
& \gamma(x)=\operatorname{card}\left\{\left(Y^{2}, \ldots, Y^{d-1}\right)\right. \text { satisfying } \\
& \left.\qquad f(x)=z_{1}, f\left(Y^{2}\right)=z_{2}, \ldots f\left(X+\sum_{i=2}^{d-1} Y^{i}\right)=z_{d}\right\}
\end{aligned}
$$


Then $\Gamma(x)=\frac{\gamma(x)}{\sum_{y} \gamma(y)}$.

$$
\begin{aligned}
\gamma(x)= & \frac{1}{2^{d}}\left[1+(-1)^{f(x)+z_{1}}\right] \times \\
& \sum_{Y^{2}} \ldots \sum_{Y^{d-1}}\left[1+(-1)^{f\left(Y^{2}\right)+z_{2}}\right]\left[1+(-1)^{f\left(Y^{d-1}\right)+z_{d-1}}\right]\left[1+(-1)^{f\left(x+{ }_{i} Y^{i}\right)+z_{d}}\right]
\end{aligned}
$$

When developing the above expression, we get:

$$
\gamma(x)=\frac{1}{2^{n+d}}\left[1+(-1)^{f(x)+z_{1}}\right]\left[2^{(d-1) n}+(-1)^{\sum_{2}^{d} z_{i}} \sum_{s}{\widehat{f_{\chi}}}^{d-1}(s)(-1)^{s . x}\right]
$$

Then

$$
\sum_{x} \gamma(x)=\frac{1}{2^{n+d}}\left[2^{d n}+(-1)^{\sum_{i=1}^{d} z_{i}} \sum_{s}{\widehat{f_{\chi}}}^{d}(s)\right]
$$

and

$$
\Gamma(x)=\left[1+(-1)^{f(x)+z_{1}}\right]^{2^{(d-1) n}+(-1)^{\sum_{2}^{d} z_{i}} \widehat{g}(x)} \begin{gathered}
2^{d n}+(-1)^{\sum_{i=1}^{d} z_{i}} \widehat{h}(0)
\end{gathered}
$$

Proposition 3 If the function $f$ is plateaued and the parity-checks are oddweighted, we have

$$
\Gamma(x)=\left[1+(-1)^{f(x)+z_{1}}\right] \begin{gathered}
2^{(d-1) n}+(-1)^{\sum_{2}^{d} z_{i}} \times 2^{(2 n-r)(p-1)} r_{f}(x) \\
2^{d n}+(-1)^{\sum_{1}^{d} z_{i}} \times 2^{(2 n-r) p+n} f_{\chi}(0)
\end{gathered}
$$

where $d=2 p+1$, and $S p\left(f_{\chi}\right)=\left\{0, \pm 2^{n-\frac{r}{2}}\right\}$

Proof. Let us recall the expression of $\Gamma(x)$ :

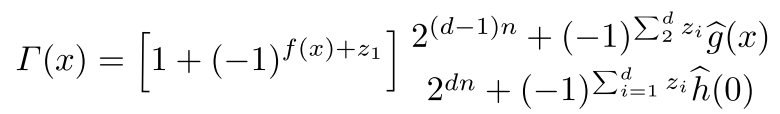

where $g={\widehat{f_{\chi}}}^{d-1}$ and $h={\widehat{f_{\chi}}}^{d}$.

If we assume that the weight of the parity-checks is odd, that is $d=2 p+1$, then

$$
\begin{aligned}
\widehat{g}(x) & =\sum_{s}{\widehat{f_{\chi}}}^{d-1}(s) \\
& =\sum_{s}{\widehat{f_{\chi}}}^{2 p}(s) \\
& =\left[2^{2 n-r}\right]^{p-1} r_{f}(x)
\end{aligned}
$$


and

$$
\begin{aligned}
\widehat{h}(0) & =\sum_{s}{\widehat{f_{\chi}}}^{d}(s) \\
& =\sum_{s}{\widehat{f_{\chi}}}^{2 p+1}(s) \\
& =\left[2^{2 n-r}\right]^{p} \times 2^{n} f_{\chi}(0)
\end{aligned}
$$

then

$$
\Gamma(x)=\left[1+(-1)^{f(x)+z_{1}}\right]^{2^{(d-1) n}+(-1)^{\sum_{2}^{d} z_{i}} \times 2^{(2 n-r)(p-1)} r_{f}(x)} \begin{gathered}
2^{d n}+(-1)^{\sum_{1}^{d} z_{i}} \times 2^{(2 n-r) p+n} f_{\chi}(0)
\end{gathered}
$$

Proposition 4 If the function is plateaued and if the parity-checks are evenweighted, we don't gain anything over the initialisation using the correlations.

Proof. If the weight of the parity-checks is even, $d=2 p$, using the same tricks as above, and using the fact that $\Gamma(x) \neq 0$ iff $f(x)=z_{1}$ iff $f_{\chi}(x)=(-1)^{z_{1}}$, we show that:

$$
\begin{aligned}
\Gamma(x) & =0 \text { if } f(x) \neq z_{1} \\
& =\frac{1}{2^{n-1}} \text { otherwise }
\end{aligned}
$$

Then, suppose $f(X(t))=1$ and recall that the Boolean function is balanced

$$
\begin{aligned}
\Lambda_{\phi_{u}}(t) & =P(u \cdot X(t)=1 \mid z, f)=\sum_{x / \phi_{u}(x)=1} \sum_{y} \prod_{E \in \mathcal{E}^{v}(t)} \Gamma_{E \in \mathcal{E}^{v}(t)}^{t} \Gamma^{t}(y) \\
& =\sum_{x / u \cdot x=1} \begin{array}{c}
\left(\frac{1}{2^{n-1}}\right)^{\mu} f(x) \\
2^{n-1} \times\left(\frac{1}{2^{n-1}}\right)^{\mu}
\end{array} \\
& =\frac{1}{2^{n-1}} \sum_{x / u \cdot x=1} f(x) \\
& =\frac{1}{2}-\frac{\widehat{f}(u)}{2^{n}}
\end{aligned}
$$

which is the probability of the equivalent BSC channel modeling the Boolean function. Therefore, when the Boolean function is plateaued, and the paritychecks even weighted, the SOJA is of no use.

Proposition 5 For any plateaued function $f$,

$$
\forall(p, q) \in \mathbb{N}, \forall v \neq 0_{n} \in\{0,1\}^{n}, \quad \sum_{s}{\widehat{f_{\chi}}}^{2 p+1}(v+s){\widehat{f_{\chi}}}^{2 q+1}(s)=0
$$


Proof. Suppose $p=0, q=0$. Then,

$$
\begin{aligned}
\sum_{s} \widehat{f_{\chi}}(v+s) \widehat{f_{\chi}}(s) & =\sum_{s} \sum_{x} \sum_{y}(-1)^{f(x)+f(y)+s \cdot(x+y)+v \cdot x} \\
& =\sum_{x} \sum_{y}(-1)^{f(x)+f(y)+v \cdot x} \times 2^{n} \mathbf{l}_{\boldsymbol{q}} x=y \diamond \\
& =2^{2 n} \delta_{0_{n}}(v) \\
& =0
\end{aligned}
$$

because $v$ is a nonzero vector.

$f$ is assumed to be a plateaued function; $\operatorname{sp}\left(f_{\chi}\right)=\{0, \pm A\}, A=2^{n-\frac{r}{2}}$

Hence,

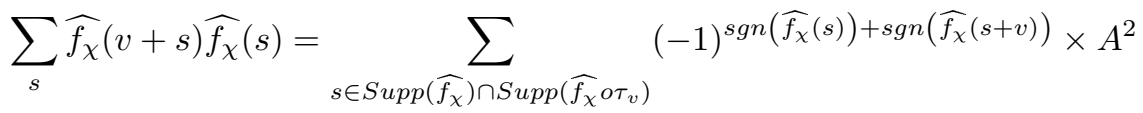

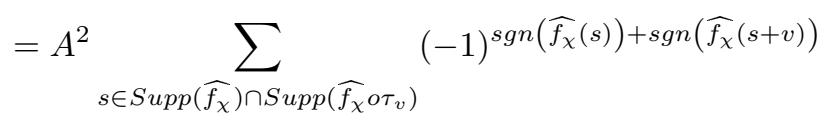

$$
\begin{aligned}
& =0
\end{aligned}
$$

Let $p, q$ be any positive integers,

$$
\begin{aligned}
& \sum_{s}{\widehat{f_{\chi}}}^{2 p+1}(v+s){\widehat{f_{\chi}}}^{2 q+1}(s) \\
= & A^{2(p+q+1)} \times \sum_{s \in \operatorname{Supp}\left({\widehat{f_{\chi}}}^{2 p+1}\right) \cap \operatorname{Supp}\left(\widehat{f}_{\chi}^{2 q+1} o \tau_{v}\right)}(-1)^{\operatorname{sgn}\left({\widehat{f_{\chi}}}^{2 p+1}(s)\right)+\operatorname{sgn}\left({\widehat{f_{\chi}}}^{2 q+1}(s+v)\right)} \\
= & 0
\end{aligned}
$$

because

$$
\begin{aligned}
\operatorname{Supp}\left({\widehat{f_{\chi}}}^{2 p+1}\right) & =\operatorname{Supp}\left(\widehat{f_{\chi}}\right) \\
\operatorname{Supp}\left({\widehat{f_{\chi}}}^{2 q+1} o \tau_{v}\right) & =\operatorname{Supp}\left(\widehat{f}_{\chi} o \tau_{v}\right) \\
\forall \operatorname{sgn}\left({\widehat{f_{\chi}}}^{2 p+1}(s)\right) & =\operatorname{sgn}\left(\widehat{f_{\chi}}(s)\right)
\end{aligned}
$$

where $\tau_{v}$ denotes the translation function:

$$
\begin{aligned}
\tau_{v}:\{0,1\}^{n} & \longrightarrow\{0,1\}^{n} \\
x & \longrightarrow x+v
\end{aligned}
$$

